\title{
Moderate and Large Deviations for the Smoothed Estimate of Sample Quantiles
}

\author{
Xiaoxia He, Xi Liu, and Chun Yao \\ College of Science, Wuhan University of Science and Technology, Wuhan 430065, China \\ Correspondence should be addressed to Xiaoxia He; hexiaoxia@wust.edu.cn
}

Received 16 February 2015; Accepted 25 May 2015

Academic Editor: Nikolaos E. Limnios

Copyright (C) 2015 Xiaoxia He et al. This is an open access article distributed under the Creative Commons Attribution License, which permits unrestricted use, distribution, and reproduction in any medium, provided the original work is properly cited.

We derive the moderate and large deviations principle for the smoothed sample quantile from a sequence of independent and identically distributed samples of size $n$.

\section{Introduction}

As it is known, the quantiles can be used for describing some properties of random variables without the restriction of moment conditions. Quantiles play a fundamental role in statistics; they are the critical values we use in hypothesis testing and interval estimation and often are the characteristics of distributions we wish most to estimate. The use of quantiles as primary measure of performance has gained prominence, particularly in microeconomic, financial, and environmental analyses and so on.

To be more specific, let $F$ denote the unknown cumulative distributions function (c.d.f.). In terms of the inverse c.d.f., the $p$-quantile is given by $\xi_{p}=F^{-1}(p)$, where

$$
F^{-1}(u)=\inf \{t ; F(t) \geq u\}, \quad u \in(0,1) .
$$

Let $F_{n}(t)$ be the empirical distributions based on the sample $\left\{X_{i} ; i=1,2, \ldots, n\right\}$; that is,

$$
F_{n}(x)=\frac{1}{n} \sum_{i=1}^{n} 1_{\left\{X_{i} \leq x\right\}}, \quad-\infty<x<\infty .
$$

Then the sample $p$-quantile based on the empirical distribution function can be represented as

$$
\widehat{\xi}_{p}=\inf \left\{x ; F_{n}(x) \geq p\right\}, \quad p \in(0,1) .
$$

The limit properties of $\widehat{\xi}_{p}$ have been studied in numerous literatures. Lahiri and Sun [1] gave Berry-Esseen theorems for samples of strongly mixing random variables under a polynomial mixing rate. $\mathrm{Wu}[2]$ established the Bahadur representation for the sample $p$-quantile for dependent sequences. Miao et al. [3] and $\mathrm{Xu}$ et al. [4] studied some asymptotic properties of the deviation between $p$-quantile and the estimator, including the moderate deviations, large deviations, and Bahadur representation. Ma et al. [5] gave the definition of sample $p$-quantile based on mid-distribution functions to provide a unified framework for asymptotic properties of sample $p$-quantile from discrete distributions.

However, $F_{n}$ does not take into account the smoothness of $F$, that is, the existence of the density function $f$. Then some investigators proposed several smoothed quantile estimates. Based on a kernel function $K$, one of the smoothed estimators for $F$ is defined as

$$
\widehat{F}_{n}(x)=\int_{\mathbb{R}} K\left(\frac{x-t}{h_{n}}\right) d F_{n}(t)=\frac{1}{n} \sum_{i=1}^{n} K\left(\frac{x-X_{i}}{h_{n}}\right),
$$

where $\left\{h_{n}\right\}$ is a positive sequence of bandwidths with $h_{n} \rightarrow 0$ as $n \rightarrow \infty$. Then, the smoothed sample quantile estimate of $\xi_{p}, \widehat{\xi}_{p n}$ is defined by

$$
\widehat{\xi}_{p n}=\widehat{F}_{n}^{-1}(p)=\inf \left\{x ; \widehat{F}_{n}(x) \geq p\right\}, \quad p \in(0,1) .
$$

Asymptotic properties for different forms of sample quantile have been investigated extensively in the literature. The kernel-type estimate of the quantile $\xi_{p}$ early work on the estimators of the quantile function includes Nadaraya [6] and 
Parzen [7]. Reiss [8] showed that the asymptotic relative deficiency of the sample quantile with respect to a linear combination of finitely many order statistics diverges to infinity as the sample size increases. Falk [9] also examined the asymptotic relative deficiency of the sample quantile compared to kernel-type quantile estimators. Yang [10] studied the asymptotic properties of kernel-type quantile estimators. Padgett [11] extended the previous works to handle right-censored data. Cai and Roussas [12] established pointwise consistency, asymptotic normality with rates, and weak convergence of the smoothed estimates.

In this paper, we will derive the pointwise moderate and large deviations principle for $\widehat{\xi}_{p n}-\xi_{p}$. There exists extensive large deviation literature involving many areas of probability and statistics. We refer to the book of Dembo and Zeitouni [13] and the references therein for an account of results and applications. In nonparametric function estimation setting, several results have been stated these last years. We refer to Louani [14], Gao [15], He and Gao [16], and Korbe Diallo and Louani [17], where results related to the kernel density estimator are obtained.

In order to state our main results, let us introduce the definition of large deviation principle. Let $(S, d)$ be a metric space and let $\left\{Y_{n}: n \geq 1\right\}$ be a sequence of $S$-valued random variables on probability space $(\Omega, \mathfrak{F}, P)$. Let $\lambda(n)$ be a sequence of positive real numbers satisfying $\lambda(n) \rightarrow \infty$ as $n \rightarrow \infty$. A function $I(\cdot): S \rightarrow[0,+\infty]$ is said to be a rate function if it is lower semicontinuous and it is said to be a good rate function if its level set $\{x \in S: I(x) \leq l\}$ is compact for all $l \geq 0$. The sequence $\left\{Y_{n}, n \geq 1\right\}$ is said to satisfy a large deviation principle with speed $\lambda(n)$ and with good rate function $I$ if, for any closed set $F$ in $S$,

$$
\limsup _{n \rightarrow \infty} \frac{1}{\lambda(n)} \log P\left(Y_{n} \in F\right) \leq-\inf _{x \in F} I(x)
$$

and, for open set $G$ in $S$,

$$
\liminf _{n \rightarrow \infty} \frac{1}{\lambda(n)} \log P\left(Y_{n} \in G\right) \leq-\inf _{x \in G} I(x) .
$$

\section{Assumptions and Main Results}

In order to display our results, we introduce some assumptions.

(A1)

$$
\lim _{x \rightarrow \infty} f(x)=0
$$

(A2) $K(x) \geq 0$, and $\int_{-\infty}^{\infty} K(x) d x=1, \int_{-\infty}^{\infty} K^{2}(x) d x<\infty$.
(A3) $I(\lambda):=\int_{-\infty}^{+\infty}(\exp \{-\lambda K(z)\}-1) d z<\infty$, for any $\lambda<0$.

(A4) $J(\lambda):=\int_{-\infty}^{+\infty} K(z) \exp \{-\lambda K(z)\} d z<\infty$, for any $\lambda<0$.

(A5) $\int_{-\infty}^{+\infty} K^{2}(z) \exp \{-\lambda K(z)\} d z<\infty$, for any $\lambda<0$.

Firstly, we give the pointwise moderate deviation principle.

Theorem 1. Let $X_{1}, X_{2}, \ldots, X_{n}$ be independent identically distributed random variables with an absolutely continuous distribution function $F(x)$, and let $\xi_{p}$ be the p-quantile of $F$ for $p \in(0,1)$. Assume that the conditions (A1) and (A2) hold; corresponding to the sample $\left\{X_{1}, X_{2}, \ldots, X_{n}\right\}$, the smoothed sample p-quantile which is denoted by $\widehat{\xi}_{p n}$ is defined as in Section 1. Let $\left\{b_{n}\right\}$ be a positive sequence satisfying

$$
\begin{aligned}
b_{n} & \longrightarrow \infty, \\
\frac{b_{n}}{\sqrt{n} h_{n}} & \longrightarrow 0 \\
\text { as } n & \longrightarrow \infty .
\end{aligned}
$$

Then, for any $r>0$, we have

$$
\begin{aligned}
\lim _{n \rightarrow \infty} \frac{1}{h_{n} b_{n}^{2}} \log P\left(\frac{\sqrt{n}}{b_{n}}\left|\widehat{\xi}_{p n}-\xi_{p}\right| \geq r\right) \\
=-\frac{r^{2}}{2 f\left(\xi_{p}\right)\left(1+\int_{-\infty}^{\infty} K^{2}(z) d z\right)} .
\end{aligned}
$$

The following result establishes a pointwise large deviation principle.

Theorem 2. Let $X_{1}, X_{2}, \ldots, X_{n}$ be independent identically distributed random variables with an absolutely continuous distribution function $F(x)$, and let $\xi_{p}$ be the p-quantile of $F$ for $p \in(0,1)$. Assume that the conditions (A1)-(A5) hold; $\widehat{\xi}_{p n}$ is defined as in Theorem 1; then, for any $r>0$, we have

$$
\begin{aligned}
& \lim _{n \rightarrow \infty} \frac{1}{n h_{n}} \log P\left(\widehat{\xi}_{p n}-\xi_{p} \geq r\right)=-\inf _{x \geq-p} \Lambda_{+}^{*}(x), \\
& \lim _{n \rightarrow \infty} \frac{1}{n h_{n}} \log P\left(\widehat{\xi}_{p n}-\xi_{p} \leq-r\right)=-\inf _{x \geq p} \Lambda_{-}^{*}(x),
\end{aligned}
$$

where

$$
\Lambda_{+}^{*}(x)= \begin{cases}J^{-1}\left(\frac{f\left(\xi_{p}+r\right)-x}{f\left(\xi_{p}+r\right)}\right)\left(x-f\left(\xi_{p}+r\right)\right)+f\left(\xi_{p}+r\right) I\left(J^{-1}\left(\frac{f\left(\xi_{p}+r\right)-x}{f\left(\xi_{p}+r\right)}\right)\right), & \text { if } x<f\left(\xi_{p}+r\right), \\ +\infty, & \text { if } x \geq f\left(\xi_{p}+r\right),\end{cases}
$$




$$
\Lambda_{-}^{*}(x)= \begin{cases}J^{-1}\left(\frac{f\left(\xi_{p}-r\right)-x}{f\left(\xi_{p}-r\right)}\right)\left(f\left(\xi_{p}+r\right)-x\right)+f\left(\xi_{p}-r\right) I\left(-J^{-1}\left(\frac{f\left(\xi_{p}-r\right)-x}{f\left(\xi_{p}-r\right)}\right)\right), & \text { if } x<f\left(\xi_{p}-r\right), \\ +\infty, & \text { if } x \geq f\left(\xi_{p}-r\right) .\end{cases}
$$

Remark 3. As it is known, whatever estimates are obtained by way of the smooth cumulative distribution function (c.d.f); they exhibit weaker rate of convergence. We can compare our moderate deviation result with that of the $\mathrm{Xu}$ and Miao [18], in which the estimation of the sample quantile was based on the c.d.f. From Theorem 1 in this paper, for $n$ large enough,

$$
P\left(\frac{\sqrt{n}}{b_{n}}\left|\widehat{\xi}_{p n}-\xi_{p}\right| \geq r\right) \approx \mathrm{e}^{-C_{1} h_{n} r^{2} b_{n}^{2}}
$$

At the same time, we can derive from $\mathrm{Xu}$ and Miao [18] that

$$
P\left(\frac{\sqrt{n}}{b_{n}}\left|\widehat{\xi}_{p n}-\xi_{p}\right| \geq r\right) \approx \mathrm{e}^{-C_{2} r^{2} b_{n}^{2}}
$$

where $C_{1}, C_{2}$ are some constants.

\section{Proof of the Main Results}

3.1. Proof of Theorem 1. For any $r>0$, we have

$$
\begin{aligned}
& P\left(\frac{\sqrt{n}}{b_{n}}\left|\widehat{\xi}_{p n}-\xi_{p}\right| \geq r\right) \\
& \quad=P\left(\widehat{\xi}_{p n} \geq \xi_{p}+\frac{b_{n}}{\sqrt{n}} r\right)+P\left(\widehat{\xi}_{p n} \leq \xi_{p}-\frac{b_{n}}{\sqrt{n}} r\right) .
\end{aligned}
$$

Then,

$$
\begin{aligned}
& P\left(\widehat{\xi}_{p n} \geq \xi_{p}+\frac{b_{n}}{\sqrt{n}} r\right) \\
& \quad=P\left(\widehat{F}_{n}\left(\xi_{p}+\frac{b_{n}}{\sqrt{n}} r\right)<p\right) \\
& \quad=P\left(\sum_{i=1}^{n}\left(V_{i n}-E V_{i n}\right) \geq n\left[(1-p)-E V_{1 n}\right]\right),
\end{aligned}
$$

where $V_{\text {in }}=1-K\left(\left(\xi_{p}+\left(b_{n} / \sqrt{n}\right) r-X_{i}\right) / h_{n}\right)$.
For any $\lambda \in \mathbb{R}$, by Taylor's expansion,

$$
\begin{aligned}
& \Lambda(\lambda):=\lim _{n \rightarrow \infty} \frac{1}{h_{n} b_{n}^{2}} \log E \\
& \cdot\left(\exp \left\{\frac{\lambda b_{n}}{\sqrt{n}} \sum_{i=1}^{n}\left(V_{\text {in }}-E V_{\text {in }}\right)\right\}\right)=\lim _{n \rightarrow \infty} \frac{1}{h_{n} b_{n}^{2}} \\
& \cdot \log \left(E\left(\exp \left\{\frac{\lambda b_{n}}{\sqrt{n}}\left(V_{\text {in }}-E V_{\text {in }}\right)\right\}\right)\right)^{n} \\
& =\lim _{n \rightarrow \infty} \frac{n}{h_{n} b_{n}^{2}} \log \left(E\left(\exp \left\{\frac{\lambda b_{n}}{\sqrt{n}}\left(V_{\text {in }}-E V_{\text {in }}\right)\right\}\right)\right)^{n} \\
& =\lim _{n \rightarrow \infty} \frac{n}{h_{n} b_{n}^{2}} \\
& \cdot \log \left(1+\frac{\lambda^{2} b_{n}^{2}}{n} \frac{E\left(V_{i n}-E V_{\text {in }}\right)^{2}}{2}+o\left(\frac{b_{n}^{2}}{n}\right)\right) .
\end{aligned}
$$

On the other hand,

$$
\begin{aligned}
E\left(V_{\text {in }}-E V_{\text {in }}\right)^{2} & \\
= & E K^{2}\left(\frac{\xi_{p}+\left(b_{n} / \sqrt{n}\right) r-X_{i}}{h_{n}}\right) \\
& -\left(E K\left(\frac{\xi_{p}+\left(b_{n} / \sqrt{n}\right) r-X_{i}}{h_{n}}\right)\right)^{2} \\
= & \int_{-\infty}^{+\infty} K^{2}\left(\frac{\xi_{p}+\left(b_{n} / \sqrt{n}\right) r-x}{h_{n}}\right) f(x) d x \\
& -\left(\int_{-\infty}^{+\infty} K\left(\frac{\xi_{p}+\left(b_{n} / \sqrt{n}\right) r-x}{h_{n}}\right) f(x) d x\right)^{2} \\
= & h_{n} \int_{-\infty}^{+\infty} K^{2}(z) f\left(\xi_{p}+\frac{b_{n}}{\sqrt{n}} r-h_{n} z\right) d z \\
& -h_{n}\left(\int_{-\infty}^{+\infty} K(z) f\left(\xi_{p}+\frac{b_{n}}{\sqrt{n}} r-h_{n} z\right) d z\right)^{2} .
\end{aligned}
$$

By Lemma 2.2 in Gao [15], we can obtain that

$$
\begin{gathered}
\lim _{n \rightarrow \infty} \frac{1}{h_{n} b_{n}^{2}} \log E\left(\exp \left\{\frac{\lambda b_{n}}{\sqrt{n}} \sum_{i=1}^{n}\left(V_{\text {in }}-E V_{\text {in }}\right)\right\}\right) \\
=\frac{\lambda^{2} f\left(\xi_{p}\right)\left(1+\int_{-\infty}^{\infty} K^{2}(z) d z\right)}{2} .
\end{gathered}
$$


Then, by Gärtner-Ellis theorem (see Dembo and Zeitouni [13]), we have

$$
\begin{gathered}
\lim _{n \rightarrow \infty} \frac{1}{h_{n} b_{n}^{2}} \log P\left(\frac{\sqrt{n}}{b_{n}}\left(\widehat{\xi}_{p n}-\xi_{p}\right) \geq r\right) \\
=-\frac{r^{2}}{2 f\left(\xi_{p}\right)\left(1+\int_{-\infty}^{\infty} K^{2}(z) d z\right)} .
\end{gathered}
$$

Likewise,

$$
\begin{aligned}
& P\left(\widehat{\xi}_{p n} \leq \xi_{p}-\frac{b_{n}}{\sqrt{n}} r\right) \\
& \quad=P\left(\widehat{F}_{n}\left(\xi_{p}-\frac{b_{n}}{\sqrt{n}} r\right) \geq p\right) \\
& \quad=P\left(\sum_{i=1}^{n}\left(Q_{i n}-E Q_{i n}\right) \geq n\left[p-E Q_{1 n}\right]\right),
\end{aligned}
$$

where $Q_{\text {in }}=K\left(\left(\xi_{p}-\left(b_{n} / \sqrt{n}\right) r-X_{i}\right) / h_{n}\right)$.

For any $\lambda \in \mathbb{R}$, by using Taylor's expansion again,

$$
\begin{gathered}
\lim _{n \rightarrow \infty} \frac{1}{h_{n} b_{n}^{2}} \log E\left(\exp \left\{\frac{\lambda b_{n}}{\sqrt{n}} \sum_{i=1}^{n}\left(Q_{\text {in }}-E Q_{\text {in }}\right)\right\}\right) \\
=\frac{\lambda^{2} f\left(\xi_{p}\right)\left(1+\int_{-\infty}^{\infty} K^{2}(z) d z\right)}{2} .
\end{gathered}
$$

Applying Gärtner-Ellis theorem, we can obtain that

$$
\begin{gathered}
\lim _{n \rightarrow \infty} \frac{1}{h_{n} b_{n}^{2}} \log P\left(\frac{\sqrt{n}}{b_{n}}\left(\widehat{\xi}_{p n}-\xi_{p}\right) \leq-r\right) \\
=-\frac{r^{2}}{2 f\left(\xi_{p}\right)\left(1+\int_{-\infty}^{\infty} K^{2}(z) d z\right)} .
\end{gathered}
$$

By (21) and (24), we can obtain the result in the theorem.
3.2. Proof of Theorem 2. For any $r>0$, by Serfling [19],

$$
\begin{aligned}
& P\left(\widehat{\xi}_{p n}-\xi_{p} \geq r\right) \\
& \quad=P\left(\widehat{F}_{n}\left(\xi_{p}+r\right)<p\right) \\
& \quad=P\left(\sum_{i=1}^{n}\left(W_{i n}-E W_{i n}\right) \geq n\left[(1-p)-E W_{1 n}\right]\right) .
\end{aligned}
$$

And, for any $\lambda \in \mathbb{R}$,

$$
\begin{aligned}
& E\left(\exp \left\{\lambda\left(W_{i n}-E W_{1 n}\right)\right\}\right) \\
& \quad=E\left(\exp \left\{\lambda\left(E\left(K\left(\frac{\xi_{p}+r-X_{i}}{h_{n}}\right)\right)-K\left(\frac{\xi_{p}+r-X_{i}}{h_{n}}\right)\right)\right\}\right) \\
& \quad=\exp \left\{\lambda h_{n} \int_{-\infty}^{\infty} K(z) f\left(\xi_{p}+r-h_{n} z\right) d z\right\} \\
& \quad \cdot h_{n} \int_{-\infty}^{+\infty} \exp \{-\lambda K(z)\} f\left(\xi_{p}+r-h_{n} z\right) d z \\
& =\exp \left\{\lambda h_{n} \int_{-\infty}^{\infty} K(z) f\left(\xi_{p}+r-h_{n} z\right) d z\right\}(1 \\
& \left.\quad+h_{n} \int_{-\infty}^{+\infty}(\exp \{-\lambda K(z)\}-1) f\left(\xi_{p}+r-h_{n} z\right) d z\right)
\end{aligned}
$$

then, by Lemma 2.2 in Gao [15],

$$
\begin{aligned}
\Lambda_{+}(\lambda):= & \lim _{n \rightarrow \infty} \frac{1}{n h_{n}} \log E\left(\exp \left\{\lambda n W_{i n}\right\}\right) \\
= & \lambda \int_{-\infty}^{\infty} K(z) f\left(\xi_{p}+r\right) d z \\
& +\int_{-\infty}^{+\infty}(\exp \{-\lambda K(z)\}-1) f\left(\xi_{p}+r\right) d z \\
= & \lambda f\left(\xi_{p}+r\right) \\
& +f\left(\xi_{p}+r\right) \int_{-\infty}^{+\infty}(\exp \{-\lambda K(z)\}-1) d z .
\end{aligned}
$$

The Fenchel-Legendre transform of $\Lambda_{+}(\lambda)$ is

$$
\begin{gathered}
\Lambda_{+}^{*}(x)=\sup _{\lambda \in \mathbb{R}}\{\lambda x-\Lambda(\lambda)\}=\sup _{\lambda \in \mathbb{R}}\left\{\lambda x-\lambda f\left(\xi_{p}+r\right)\right. \\
\left.-f\left(\xi_{p}+r\right) \int_{-\infty}^{+\infty}(\exp \{-\lambda K(z)\}-1) d z\right\} .
\end{gathered}
$$

By simple calculation, we can obtain

$$
\Lambda_{+}^{*}(x)= \begin{cases}J^{-1}\left(\frac{f\left(\xi_{p}+r\right)-x}{f\left(\xi_{p}+r\right)}\right)\left(x-f\left(\xi_{p}+r\right)\right)+f\left(\xi_{p}+r\right) I\left(J^{-1}\left(\frac{f\left(\xi_{p}+r\right)-x}{f\left(\xi_{p}+r\right)}\right)\right), & \text { if } x<f\left(\xi_{p}+r\right) \\ +\infty & \text { if } x \geq f\left(\xi_{p}+r\right) .\end{cases}
$$


Then, by the Cramér theorem (see Dembo and Zeitouni [13]), we have

$$
\begin{aligned}
& \lim _{n \rightarrow \infty} \frac{1}{n h_{n}} \log P\left(\widehat{\xi}_{p n}-\xi_{p} \geq r\right)=\lim _{n \rightarrow \infty} \frac{1}{n h_{n}} \log P \\
& \cdot\left(\sum_{i=1}^{n}\left(W_{i n}-E W_{i n}\right) \geq n\left[(1-p)-E W_{1 n}\right]\right) \\
& =-\inf _{x \geq-p} \Lambda_{+}^{*}(x) .
\end{aligned}
$$

Similarly,

$$
\begin{aligned}
& P\left(\widehat{\xi}_{p n}-\xi_{p} \leq-r\right) \\
& \quad=P\left(\widehat{F}_{n}\left(\xi_{p}-r\right) \geq p\right) \\
& \quad=P\left(\sum_{i=1}^{n}\left(U_{i n}-E U_{i n}\right) \geq n\left[p-E U_{1 n}\right]\right) .
\end{aligned}
$$

$$
\Lambda_{-}^{*}(x)= \begin{cases}J^{-1}\left(\frac{f\left(\xi_{p}-r\right)-x}{f\left(\xi_{p}-r\right)}\right)\left(f\left(\xi_{p}+r\right)-x\right)+f\left(\xi_{p}-r\right) I\left(-J^{-1}\left(\frac{f\left(\xi_{p}-r\right)-x}{f\left(\xi_{p}-r\right)}\right)\right), & \text { if } x<f\left(\xi_{p}-r\right), \\ +\infty, & \text { if } x \geq f\left(\xi_{p}-r\right) .\end{cases}
$$

And, for any $\lambda \in \mathbb{R}$,

$$
\begin{aligned}
E & \left(\exp \left\{\lambda\left(U_{i n}-E U_{1 n}\right)\right\}\right) \\
& =E\left(\exp \left\{\lambda\left(K\left(\frac{\xi_{p}-r-X_{i}}{h_{n}}\right)-E\left(K\left(\frac{\xi_{p}-r-X_{i}}{h_{n}}\right)\right)\right)\right\}\right) \\
& =\exp \left\{-\lambda h_{n} \int_{-\infty}^{\infty} K(z) f\left(\xi_{p}-r-h_{n} z\right) d z\right\} \\
& \cdot h_{n} \int_{-\infty}^{+\infty} \exp \{\lambda K(z)\} f\left(\xi_{p}-r-h_{n} z\right) d z \\
& =\exp \left\{-\lambda h_{n} \int_{-\infty}^{\infty} K(z) f\left(\xi_{p}-r-h_{n} z\right) d z\right\}(1 \\
& \left.+h_{n} \int_{-\infty}^{+\infty}(\exp \{\lambda K(z)\}-1) f\left(\xi_{p}-r-h_{n} z\right) d z\right) .
\end{aligned}
$$

Then,

$$
\begin{aligned}
\Lambda_{-}(\lambda):= & \lim _{n \rightarrow \infty} \frac{1}{n h_{n}} \log E\left(\exp \left\{\lambda n U_{i n}\right\}\right) \\
= & \lambda f\left(\xi_{p}-r\right) \\
& +f\left(\xi_{p}-r\right) \int_{-\infty}^{+\infty}(\exp \{\lambda K(z)\}-1) d z .
\end{aligned}
$$

The Fenchel-Legendre transform of $\Lambda_{-}(\lambda)$ is
Then, we obtain (12), and we complete the proof of Theorem 2.

\section{Conflict of Interests}

The authors declare that there is no conflict of interests regarding the publication of this paper.

\section{Acknowledgments}

This work is partly supported by the National Natural Science Foundation of China (no. 11201356) and the Hubei Province Key Laboratory of Systems Science in Metallurgical Process (Wuhan University of Science and Technology) (no. Y201306).

\section{References}

[1] S. N. Lahiri and S. Sun, "A Berry-Esseen theorem for sample quantiles under weak dependence," The Annals of Applied Probability, vol. 19, no. 1, pp. 108-126, 2009.

[2] W. B. Wu, "On the Bahadur representation of sample quantiles for dependent sequences," The Annals of Statistics, vol. 33, no. 4, pp. 1934-1963, 2005.
[3] Y. Miao, Y.-X. Chen, and S.-F. Xu, "Asymptotic properties of the deviation between order statistics and $p$-Quantile," Communications in Statistics - Theory and Methods, vol. 40, no. 1, pp. 8-14, 2011.

[4] S. F. Xu, L. Ge, and Y. Miao, "On the Bahadur representation of sample quantiles and order statistics for NA sequences," Journal of the Korean Statistical Society, vol. 42, no. 1, pp. 1-7, 2013.

[5] Y. Ma, M. G. Genton, and E. Parzen, "Asymptotic properties of sample quantiles of discrete distributions," Annals of the Institute of Statistical Mathematics, vol. 63, no. 2, pp. 227-243, 2011.

[6] E. A. Nadaraya, "Some new estimates for distribution function," Theory of Probability and Its Applications, vol. 9, pp. 497-500, 1964.

[7] E. Parzen, "Nonparametric statistical data modeling," Journal of the American Statistical Association, vol. 74, no. 365, pp. 105-131, 1979.

[8] R.-D. Reiss, "Estimation of quantiles in certain nonparametric models," The Annals of Statistics, vol. 8, no. 1, pp. 87-105, 1980.

[9] M. Falk, "Relative deficiency of kernel type estimators of quantiles," The Annals of Statistics, vol. 12, no. 1, pp. 261-268, 1984.

[10] S.-S. Yang, "A smooth nonparametric estimator of a quantile function," Journal of the American Statistical Association, vol. 80, no. 392, pp. 1004-1011, 1985.

[11] W. J. Padgett, "A kernel-type estimator of a quantile function from right-censored data," Journal of the American Statistical Association, vol. 81, no. 393, pp. 215-222, 1986. 
[12] Z. Cai and G. G. Roussas, "Smooth estimate of quantiles under association," Statistics \& Probability Letters, vol. 36, no. 3, pp. 275-287, 1997.

[13] A. Dembo and O. Zeitouni, Large Deviations Techniques and Applications, vol. 38 of Applications of Mathematics (New York), Springer, New York, NY, USA, 2nd edition, 1998.

[14] D. Louani, "Large deviations limit theorems for the kernel density estimator," Scandinavian Journal of Statistics. Theory and Applications, vol. 25, no. 1, pp. 243-253, 1998.

[15] F. Gao, "Moderate deviations and large deviations for kernel density estimators," Journal of Theoretical Probability, vol. 16, no. 2, pp. 401-418, 2003.

[16] X. He and F. Gao, "Moderate deviations and large deviations for a test of symmetry based on kernel density estimator," Acta Mathematica Scientia. Series B. English Edition, vol. 28, no. 3, pp. 665-674, 2008.

[17] A. O. Korbe Diallo and D. Louani, "Moderate and large deviation principles for the hazard rate function kernel estimator under censoring," Statistics \& Probability Letters, vol. 83, no. 3, pp. 735-743, 2013.

[18] S. F. Xu and Y. Miao, "Limit behaviors of the deviation between the sample quantiles and the quantile," Filomat, vol. 25, no. 2, pp. 197-206, 2011.

[19] R. J. Serfling, Approximation Theorems of Mathematical Statistics, John Wiley \& Sons, New York, NY, USA, 1980. 


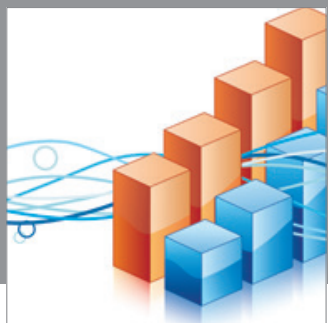

Advances in

Operations Research

mansans

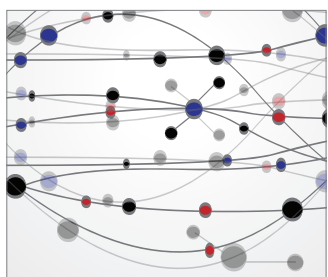

The Scientific World Journal
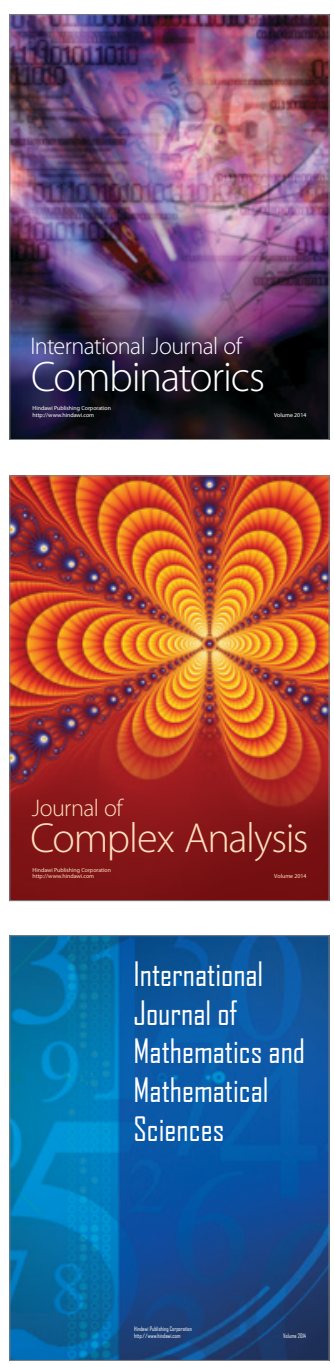
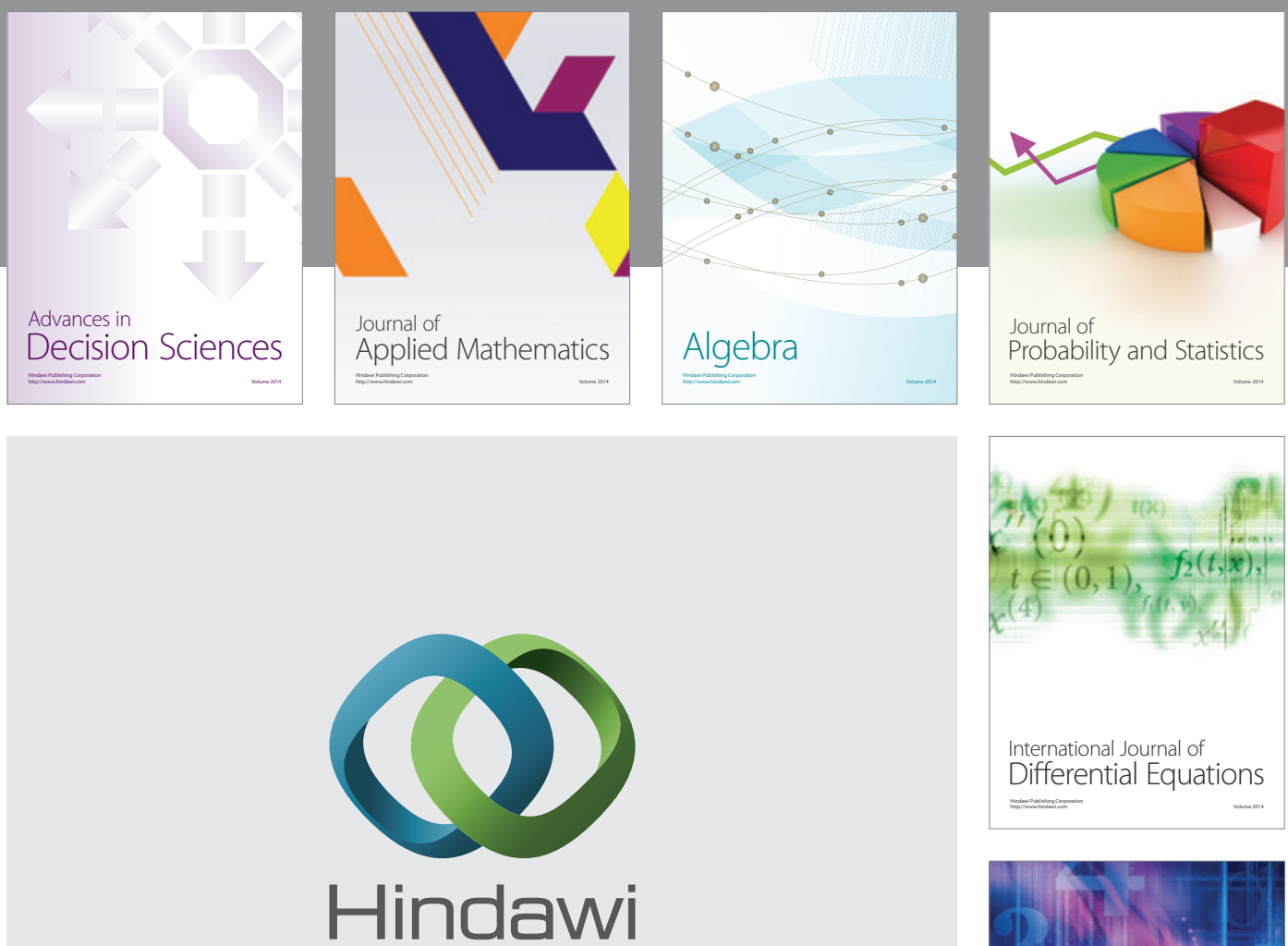

Submit your manuscripts at http://www.hindawi.com
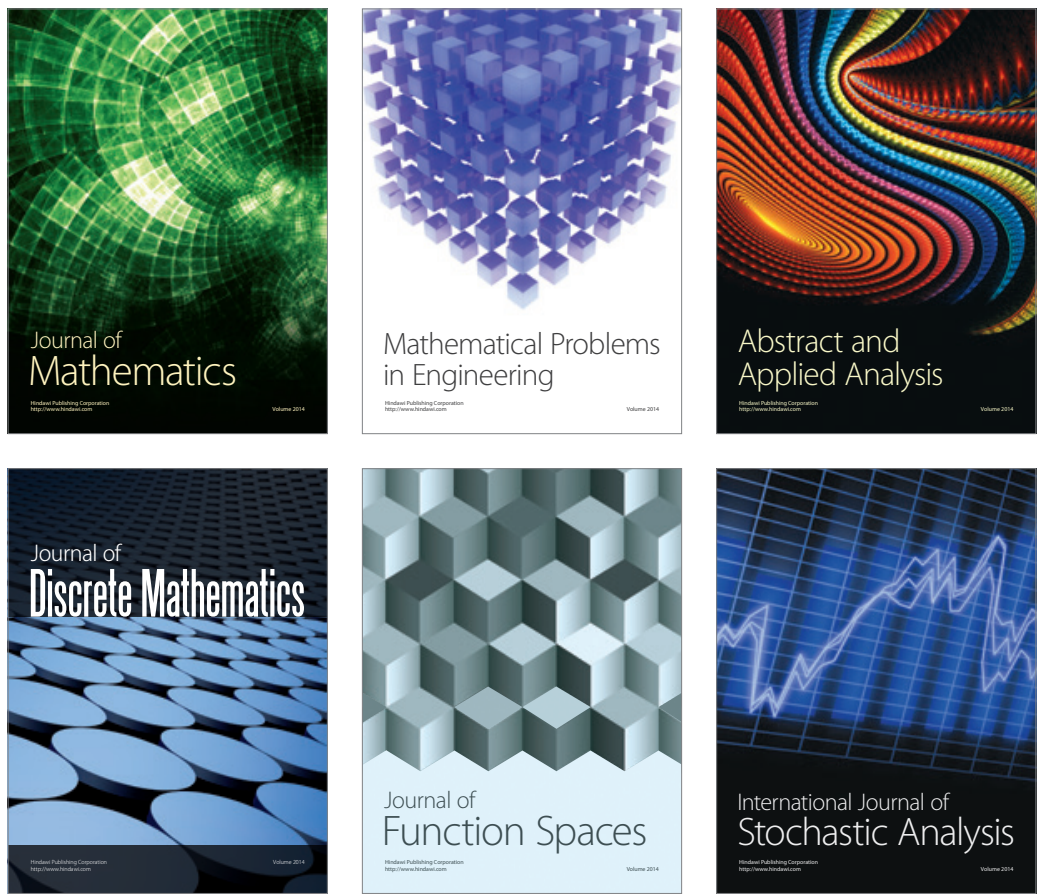

Journal of

Function Spaces

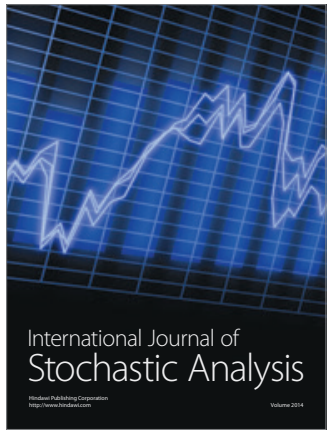

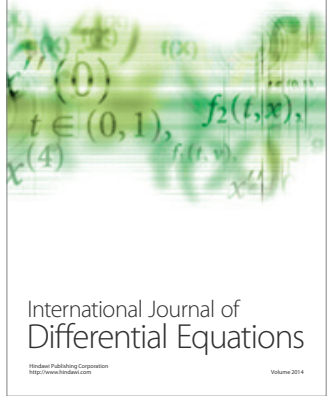
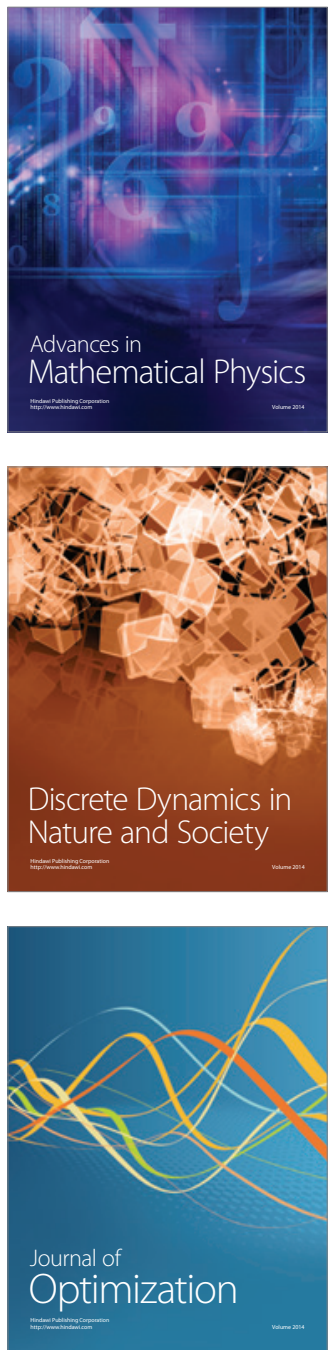